\title{
ASYMPTOTIC SOLUTION OF A CRACK IN A VANISHINGLY THIN ELLIPTIC INHOMOGENEITY
}

\author{
BY \\ CHIEN H. WU \\ University of Illinois at Chicago, Illinois
}

\begin{abstract}
When a crack of finite size is wholly surrounded by a thin inhomogeneity, the behavior of the solution near a crack tip is the same as that of a semi-infinite crack surrounded by a semi-infinite inhomogeneity. The analytical structure of the solution for the latter problem is established via the consideration of the title problem. It is shown that the standard Fast Fourier Transform Algorithm may be applied for the determination of the coefficients involved in the analytically structured series solution. An approximate but explicit solution is also derived for the title problem.
\end{abstract}

1. Introduction. When a crack is wholly embedded in an inhomogeneity or when the crack tips are separately lodged in disjointed inhomogeneities, differences between the moduli of the inhomogeneity and the matrix material can cause the SIF to be greater or less than that prevailing in a homogeneous body. With the problem of crack-damage interaction in mind, the inhomogeneities are taken to be vanishingly small and softer than the matrix. This is the range in which we place our emphasis.

For a finite crack with tips lodged in vanishingly small tip inhomogeneities, the asymptotic limit is the solution for a semi-infinite crack lodged in an inhomogeneity of finite size. The case of a semi-infinite crack penetrating a circular inhomogeneity was studied by Steif [5]. A method for solving noncircular cases was considered by Hutchinson [4]. Using Eshelby tensor (Eshelby, [3]) to estimate the Eshelby-tensor force along the interface, an approximate but explicit formula was obtained by $\mathrm{Wu}$ [7]. If the crack is of linear dimension unity and the size of the inhomogeneity is characterized by $\varepsilon \quad(\varepsilon \rightarrow 0)$, asymptotic solutions accurate to the order of $\varepsilon$ may be constructed for arbitrary crack-specimen configuration and inhomogeneity shape. This result, together with the associated Eshelby-tensor force, is being completed by us.

For the cases where the crack is wholly embedded in an inhomogeneity the needed asymptotic analysis is completely different, as the matrix contains no portion of the traction-free boundary. This last condition dictates the holomorphicity of the solution for the matrix for the class of problems described in the foregoing paragraph. Without the direct availability of such information, the properties of the solution for

Received March 14, 1989.

(C)1990 Brown University 
the matrix can only be established from matching. The situation parallels to that associated with the thin airfoil problem (Van Dyke [6]) except that the "airfoil" now is also governed by a field. In fact the field is singular due to the presence of a crack inside the "airfoil." Moreover, both the "airfoil" and the outside field are each governed by two complex functions. The complexity of the problem makes it clear that the desired asymptotic limit can only be obtained by numerical means. The actual computation, however, must be preceded by an asymptotic analysis to get rid of the thinness parameter $\varepsilon$. This is necessary because no numerical scheme can possibly handle the conflicting limits required by $\varepsilon \rightarrow 0$ (vanishingly thin inhomogeneity) and $r^{-1 / 2} \rightarrow \infty$ (crack tip inside the inhomogeneity).

Our eventual goal is to improvise an asymptotic analysis, together with a computational scheme, that is applicable to arbitrary crack-specimen configuration and inhomogeneity shape. A number of underlying difficulties inherent to the goal are either resolved or made apparent by the explicit analysis completed for the benchmark problem presented in this paper.

The title problem is a benchmark problem for a number of reasons. The confocal geometry enables us to represent the full solution for arbitrary inhomogeneity size in series form in a transformed plane so that the numerically exact result may be considered as a checkpoint. This was completed by $\mathrm{Wu}$ and $\mathrm{Chen} \mathrm{[8],} \mathrm{and} \mathrm{the} \mathrm{de-}$ sired asymptotic limit was numerically extrapolated. The series solution, however, does not reveal any of the analytic characters of the solution around the crack-tip region that is immediately outside the inhomogeneity. It merely indicates that the convergence of the series becomes extremely slow when the size of the inhomogeneity becomes vanishingly small. This missing information is completely recovered in the asymptotic analysis. In particular, the solution for the matrix in the neighborhood immediately next to the crack tip must involve powers of $r^{-1 / 2}$ where $r=0$ is the crack tip. This is in contrast to the crack-tip inhomogeneity solution where the series is of the form of $r^{-(n+1 / 2)}$.

Section 2 summarizes the formulation in terms of a complex variable. It is shown that the solution depends explicitly on two composite parameters $\gamma$ and $\gamma^{*}$, which may be expressed in terms of Dundurs' [1] $\alpha$ and $\beta$ parameter. While the admissible elasticity ranges can be most easily determined in the $\alpha \beta$-plane, the explicit dependence of the solution on $\gamma$ and $\gamma^{*}$ suggests that solutions for similar inhomogeneity problems should be presented in terms of $\gamma$ and $\gamma^{*}$ rather than other equally valid parameters.

Outer expansions are presented in Sec. 3. The procedure follows straightforwardly from the well-known thin airfoil expansion (Van Dyke [6]). The needed asymptotic sequence is a part of the unknown in a typical asymptotic analysis. For example, if the inhomogeneity is a thin strip of thickness $2 \varepsilon$ with round noses of radius $\varepsilon$ the asymptotic sequence must include $\varepsilon \ln \varepsilon$. Thus, the form of an outer expansion depends explicitly on the shape of the inhomogeneity.

Inner expansion is presented in Sec. 4, and the parabolic nose is studied in detail. Forms of the appropriate series representations are obtained. It is shown that the standard Fast Fourier Transform Algorithm may be applied to the system. For 
the parabolic nose, a two-term approximate but explicit solution is also presented. Pertinent results are presented in Sec. 5. The author is indebted to Mr. C. H. Chen for many helpful discussions and several lengthy computations without which the concise graphic presentation summarized in Sec. 5 would have been impossible.

2. Complex variable formulation. Let $\left(z_{1}, z_{2}\right)$ be rectangular Cartesian coordinates, and $z=z_{1}+i z_{2}$ the associated complex variable in the $z$-plane. For plane problems, the displacements $u_{\alpha}\left(z_{1}, z_{2}\right)$, stresses $\tau_{\alpha \beta}\left(z_{1}, z_{2}\right)$ and resultant force over an arc $R=R_{1}+i R_{2}$ may be expressed in terms of two complex functions $W(z)$ and $w(z)$, viz.,

where

$$
\begin{gathered}
2 \mu\left(u_{1}+i u_{2}\right)=\kappa W(z)-z \overline{W^{\prime}(z)}-\overline{w(z)}, \\
i R=W(z)+z \overline{W^{\prime}(z)}+\overline{w(z)},
\end{gathered}
$$

$$
\kappa= \begin{cases}3-4 \nu & \text { plane stress, } \\ (3-\nu) /(1+\nu) & \text { plane strain, }\end{cases}
$$

and $\mu$ and $\nu$ are, respectively, shear modulus and Poisson's ratio.

For regions containing a portion of the real axis along which displacement and traction are continuous, the function $w(z)$ may be expressed in terms of $W(z)$ and a new function $f(z)$ as follows (England [2]):

$$
w(z)=\overline{W(\bar{z})}-z W^{\prime}(z)-\overline{f(\bar{z})} .
$$

Using the above, we obtain from (2.1) and (2.2)

$$
\begin{gathered}
i R=W(z)+W(\bar{z})+(z-\bar{z}) \overline{W^{\prime}(z)}-f(\bar{z}), \\
2 \mu\left(u_{1}+i u_{2}\right)=(\kappa+1) W(z)-W(z)+W(\bar{z})+(z-\bar{z}) \overline{W^{\prime}(z)}-f(\bar{z}) .
\end{gathered}
$$

A traction-free crack of length 2 is located on the real axis with $\left|z_{1}\right|<1$. The crack is wholly embedded in an inhomogeneity, denoted by $D_{1}$, which in turn is embedded in an infinite medium denoted by $D_{2}$, Fig. 1a. An additional subscript $\alpha$ will be placed on a parameter or variable to indicate its region of definition. Thus, $\mu_{\alpha}, \nu_{\alpha}, \kappa_{\alpha}, W_{\alpha}$, and $f_{\alpha}$ are defined for region $D_{\alpha}$.

The crack is located in $D_{1}$ and the associated traction-free condition may be integrated once to become (c.f. (2.5))

$$
W_{1}^{ \pm}(x)+W_{1}^{\mp}(x)-f_{1}^{\mp}(x)=0 \quad(|x|<1)
$$

where the notation $F^{ \pm}(x)=F(x \pm i 0)$ has been used.

The infinite medium is loaded at infinity by $\tau_{\alpha \beta}=\sigma_{\alpha \beta}$ so that

$$
W_{2}=W z, \quad f_{2}=f z \quad \text { as } z \rightarrow \infty
$$

where

and

$$
W=\frac{1}{4}\left(\sigma_{11}+\sigma_{22}\right), \quad f=\frac{1}{2}\left(\sigma_{11}-\sigma_{22}\right)+i \sigma_{12},
$$

$$
\sigma=2 W-f=\sigma_{22}-i \sigma_{12}
$$

is another parameter to be used in the sequel. 


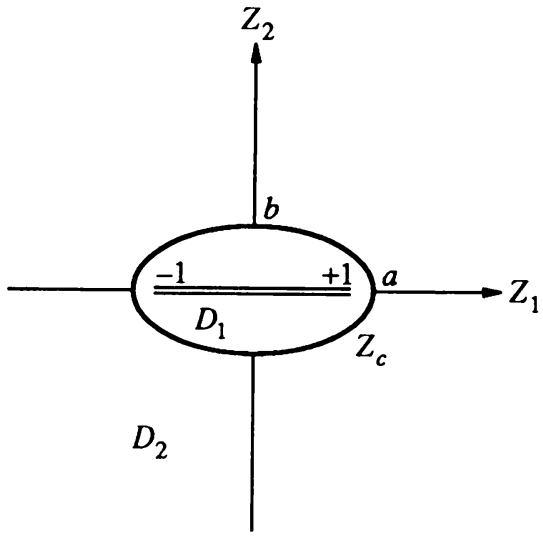

(a)

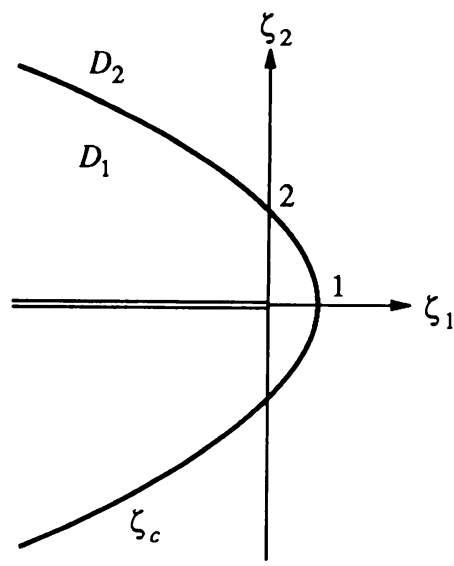

(b)

FIG. 1. A crack in a vanishingly thin elliptic inhomogeneity. (a) configuration in the physical $z$-plane, $b / a \rightarrow 0$. (b) Parabolic nose in the boundary layer $\zeta$-plane.

The interface between $D_{1}$ and $D_{2}$ is denoted by $C$ and is defined by

$$
C: z=z_{i} \text {. }
$$

It is assumed that $C$ is perfectly bonded so that traction and displacement are continuous along $C$. In particular, the traction continuity condition may be integrated once to become a continuity condition in $R$ (c.f. (2.5)). The two conditions are

$$
\begin{gathered}
W_{1}\left(z_{c}\right)+W_{1}\left(\bar{z}_{c}\right)+\left(z_{c}-\bar{z}_{c}\right) \overline{W_{1}^{\prime}\left(z_{c}\right)}-f_{1}\left(\bar{z}_{c}\right) \\
=W_{2}\left(z_{c}\right)+W_{2}\left(\bar{z}_{c}\right)+\left(z_{c}-\bar{z}_{c}\right) \overline{W_{2}^{\prime}\left(z_{c}\right)}-f_{2}\left(\bar{z}_{c}\right), \\
W_{1}\left(z_{c}\right)=\gamma W_{2}\left(z_{2}\right)+\gamma^{*}\left[W_{2}\left(z_{c}\right)+W_{2}\left(\bar{z}_{c}\right)+\left(z_{c}-\bar{z}_{c}\right) \overline{W_{2}^{\prime}\left(z_{c}\right)}-f_{c}\left(\bar{z}_{c}\right)\right],
\end{gathered}
$$

where the $R$-continuity, (2.12), has been used in simplifying the displacement continuity conditions, (2.13), and

$$
\gamma=\frac{\left(1+\kappa_{2}\right) \mu_{1}}{\left(1+\kappa_{1}\right) \mu_{2}}, \quad \gamma^{*}=\frac{1}{1+\kappa_{1}}\left(1-\frac{\mu_{1}}{\mu_{2}}\right),
$$

are two composite parameters. A discussion of composite parameters may be found in (Dundurs [1]). Equations (2.7), (2.8), (2.12), and (2.13) constitute the governing conditions for the solution of the desired problem. Attention is now turned to the specific situation where the interface $C$ is a thin ellipse.

We begin by specifying the following parameters (Fig. 1a):

$$
\rho_{c}=1+\varepsilon, \quad a=\frac{1}{2}\left(\rho_{c}+\frac{1}{\rho_{c}}\right), \quad b=\frac{1}{2}\left(\rho_{c}-\frac{1}{\rho_{c}}\right), \quad a^{2}-b^{2}=1,
$$


where $a$ and $b$ are the major and minor axes of an ellipse with focal points located at \pm 1 . The ellipse is thin if $\varepsilon \ll 1$. We have

$$
\begin{gathered}
a=1+\frac{1}{2}\left(\varepsilon^{2}-\varepsilon^{3}+\cdots\right), \quad b=\varepsilon-\frac{1}{2}\left(\varepsilon^{2}-\varepsilon^{3}+\cdots\right), \\
\delta(\varepsilon)=b / a \sim O(\varepsilon)
\end{gathered}
$$

where $\delta$ is another convenient parameter. Moreover, the radius of curvature of the ellipse at $z= \pm a$ is

$$
\rho_{a}=\frac{b^{2}}{a} \sim \delta^{2}+O\left(\varepsilon^{4}\right) .
$$

The ellipse is assumed to be the interface boundary, viz.,

$$
C: z=z_{c}=x \pm i \frac{b}{a}\left(a^{2}-x^{2}\right)^{1 / 2}, \quad\left(x^{2}<a^{2}\right) .
$$

Thus, as $\varepsilon \rightarrow 0, z_{c}$ has the expansion

$$
z_{c} \sim x \pm i \delta\left(1-x^{2}\right)^{1 / 2} \pm i \delta^{3} \frac{1}{2}\left(1-x^{2}\right)^{-1 / 2}+\cdots
$$

which is valid for $x^{2}<1$. It follows that the expansion cannot be used to satisfy the continuity near $z= \pm a$. The associated expansion is termed the outer expansion which will be presented in Sec. 3. The terminology has its origin in thin airfoil theory which is well known [6].

To remedy the shortcoming of the outer expansion, inner expansions will be constructed in Sec. 4. In view of the symmetry, we shall concentrate on the region near $z=a$. The scaling factor for the needed boundary-layer complex variable is dictated by (2.16). This complex variable is defined by (Fig. 1b)

$$
\zeta=(z-1) / \frac{1}{2} \delta^{2}
$$

The appropriate portion of $C$ is now given by the expansion:

$$
C: \zeta \sim \zeta_{c} \sim \xi \pm i 2\left[(1-\xi)-\frac{\delta^{2}}{4}(1-\xi)^{2}+\cdots\right]^{1 / 2}
$$

where $\xi<1$ and the leading term is just the parabola:

$$
\begin{array}{rlrl}
\zeta_{c} \sim \zeta_{0} & =\left(1-\eta^{2}\right)+i 2 \eta & (-\infty<\eta<+\infty), \\
& =\left(\cos \frac{\phi}{2}\right)^{-2} e^{i \phi} \quad(-\pi<\phi<\pi) .
\end{array}
$$

3. Outer expansion. The representation (2.20) will be used to satisfy (2.12) and (2.13). In view of (2.17) and (2.20), we choose $\delta^{n}$ as the asymptotic sequence and seek the solution of a generic unknown function $F(z)$ in the form

$$
F(z) \sim F(z, \delta) \sim \sum_{n=0}^{\infty} \delta^{n} F_{n}(z)
$$


where $F$ stands for any of the four unknown functions $W_{\alpha}$ and $f_{\alpha}$. The value of the function $F\left(z_{c}\right)$ may be computed from the scheme:

$$
\begin{aligned}
F\left(z_{c}\right) & \sim \sum \delta^{n} F_{n}\left(z_{c}\right) \\
& \sim \sum \delta^{n}\left[F_{n}^{ \pm}(x) \pm i \delta\left(1-x^{2}\right)^{1 / 2} F_{n}^{\prime \pm}(x)+\cdots\right]
\end{aligned}
$$

where (2.20) has been used for $z_{c}$.

The system of governing conditions (2.7), (2.12), (2.13), and (2.8) are now expanded in accordance with (3.1) and (3.2). The $\delta^{0}$-terms are:

$$
\begin{gathered}
W_{10}^{ \pm}(x)+W_{10}^{\mp}(x)-f_{10}^{\mp}(x)=0, \\
W_{20}^{ \pm}(x)+W_{20}^{\mp}(x)-f_{20}^{\mp}(x)=W_{10}^{ \pm}(x)+W_{10}^{\mp}(x)-f_{10}^{\mp}(x), \\
W_{10}^{ \pm}(x)=\gamma W_{20}^{ \pm}(x)+\gamma^{*}\left[W_{20}^{ \pm}(x)+W_{20}^{\mp}(x)-f_{20}^{\mp}(x)\right], \\
W_{20}(z)=W z, \quad f_{20}(z)=f z \quad \text { as } z \rightarrow \infty,
\end{gathered}
$$

where the first three conditions apply to the interval $|x|<1$. It follows from (3.3) that the left-hand side of (3.4) vanishes as well. It merely implies that when an interface is asymptotically near a traction-free boundary it is itself asymptotically traction-free. This is essentially the nature of the iteration associated with the outer expansion. At the same time, it is clear that the iterative mechanism cannot be valid near $z= \pm a$ where the interface traction is asymptotically large in $(z-1)^{-1 / 2}$ as $z=a \rightarrow 1$. In any case the solution to the above equations is

$$
\begin{gathered}
f_{10}(z)=\gamma f_{20}(z)=\gamma f z, \\
2 W_{10}(z)-f_{10}(z)=\gamma\left[2 W_{20}(z)-f_{20}(z)\right]=\gamma \sigma X(z),
\end{gathered}
$$

where

$$
X(z)=\left(z^{2}-1\right)^{1 / 2} \text {. }
$$

The $\delta$-terms of the system of equations are then derived. They may be further simplified by the use of (3.7) and (3.8). The results are

$$
\begin{gathered}
W_{11}^{ \pm}(x)+W_{11}^{\mp}(x)-f_{11}^{\mp}(x)=0 \\
W_{21}^{ \pm}(x)+W_{21}^{\mp}(x)-f_{21}^{\mp}(x)=(\gamma-1)\left[(\sigma-\bar{\sigma}) x \pm(f+\bar{f}) i\left(1-x^{2}\right)^{1 / 2}\right], \\
W_{11}^{ \pm}(x)=\gamma W_{21}^{ \pm}(x)+\gamma \gamma^{*}\left[(\sigma-\bar{\sigma}) x \pm(f+\bar{f}) i\left(1-x^{2}\right)^{1 / 2}\right], \\
W_{21}(z) \text { and } f_{21}(z) \rightarrow 0 \text { as } z \rightarrow \infty .
\end{gathered}
$$

Combining the \pm forms of (3.11), we obtain

$$
\begin{gathered}
f_{21}^{+}(x)-f_{21}^{-}(x)=i 2(\gamma-1)(f+\bar{f})\left(1-x^{2}\right)^{1 / 2}, \\
{\left[2 W_{21}^{+}(x)-f_{21}^{+}(x)\right]+\left[2 W_{21}^{-}(x)-f_{21}^{-}(x)\right]=2(\sigma-\bar{\sigma})(\gamma-1) x,}
\end{gathered}
$$

which, together with (3.13), may be solved for $f_{21}$ and $W_{21}$ in terms of a Cauchy and a Hilbert problem. The solutions are:

$$
\begin{gathered}
f_{21}(z)=(\gamma-1)(f+\bar{f})[X(z)-z], \\
2 W_{21}(z)-f_{21}(z)=-(\gamma-1)(\sigma-\bar{\sigma})[X(z)-z],
\end{gathered}
$$


where $z$ is a homogeneous solution of (3.14) and $X(z)$ a homogeneous solution of (3.15). They are included to meet the conditions at infinity. Substituting the above into (3.12) and (3.10), we get

$$
\begin{gathered}
f_{11}(z)=\gamma\left[\left(\gamma-1+2 \gamma^{*}\right)(\sigma-\bar{\sigma})-(\gamma-1)(f+\bar{f})\right] z, \\
2 W_{11}(z)-f_{11}(z)=\gamma\left[\left(\gamma-1+2 \gamma^{*}\right)(f+\bar{f})-(\gamma-1)(\sigma-\bar{\sigma})\right] X(z) .
\end{gathered}
$$

The $\delta^{2}$-terms of the system of governing conditions are obtained in a similar manner. They are further simplified by the explicit lower order solutions and the results are:

$$
\begin{gathered}
W_{12}^{ \pm}(x)+W_{12}^{\mp}(x)-f_{12}^{\mp}(x)=0, \\
W_{22}^{ \pm}(x)+W_{22}^{\mp}(x)-f_{22}^{\mp}(x)=(\gamma-1)[(2 \gamma-1) f-(2 \gamma+3) \bar{f}] x \\
\mp(\gamma-1)[(2 \gamma-1) f+(2 \gamma-3) \bar{f}] i\left(1-x^{2}\right)^{1 / 2} \\
\quad-\frac{(\gamma-1) \bar{\sigma}}{ \pm i\left(1-x^{2}\right)^{1 / 2}}, \\
W_{12}^{ \pm}(x)=\gamma W_{22}^{ \pm}(x)-\gamma \gamma^{*}\left[(f+\bar{f}) x \pm(\sigma-\bar{\sigma}) i\left(1-x^{2}\right)^{1 / 2}\right] \\
+\gamma \gamma^{*}\left[2(\gamma-1)(f+\bar{f}) x \mp 2(\gamma-1)(f+\bar{f}) i\left(1-x^{2}\right)^{1 / 2}\right. \\
\left.-\frac{\bar{\sigma}}{\left. \pm i\left(1-x^{2}\right)^{1 / 2}\right]}\right] \\
W_{22}(z) \text { and } f_{22}(z) \rightarrow 0 \text { as } z \rightarrow \infty .
\end{gathered}
$$

Combining the \pm forms of (3.21) again leads to a Cauchy and a Hilbert problem, and the solutions are

$$
\begin{aligned}
f_{22}(z)=- & (\gamma-1)[(2 \gamma-1) f+(2 \gamma-3) \bar{f}][X(z)-z] \\
-(\gamma-1) \bar{\sigma} X^{-1}(z) & \\
2 W_{22}(z)-f_{22}(z)= & (\gamma-1)[(2 \gamma+3) \bar{f}-(2 \gamma-1) f][X(z)-z] \\
& +C(\gamma-1) \bar{\sigma} X^{-1}(z)
\end{aligned}
$$

where the last term of (3.25) is a homogeneous solution and $C$ an arbitrary constant. The other two functions may again be determined from (3.22) and (3.20). They are

$$
\begin{aligned}
f_{12}(z)=2\{-\gamma(\gamma-1)[3 \bar{f}- & \left.(2 \gamma-1) f]-\gamma \gamma^{*}(f+\bar{f})+2 \gamma \gamma^{*}(\gamma-1)(f-\bar{f})\right\} z, \\
2 W_{12}(z)-f_{12}(z)= & \left.(C-1)(\gamma-1)-2 \gamma^{*}\right] \gamma \bar{\sigma} X^{-1}(z) \\
& +2\left\{\gamma(\gamma-1)\left[3 \bar{f}-(2 \gamma-1) f-\gamma \gamma^{*}(\sigma-\bar{\sigma})\right]\right. \\
& \left.-2 \gamma \gamma^{*}(\gamma-1)(f+\bar{f})\right\} X(z) .
\end{aligned}
$$

The procedure could be continued to include as many terms as we wish but the explicit 3-term expansion is sufficient to reveal the most important characters of the desired solution. To unveil these properties and also to facilitate matching, we need 
the inner expansion of the outer expansion. This is accomplished by expressing $z$ in terms of $\zeta$ via (2.21) and then expanding. The results are

$$
\begin{gathered}
f_{1} \sim \gamma f+\delta\left\{\gamma\left[(1-\gamma)(f+\bar{f})-\left(1-\gamma-2 \gamma^{*}\right)(\sigma-\bar{\sigma})\right]+\sum_{n=1}^{\infty} \frac{()_{n}}{\zeta^{n}}\right\}+O\left(\delta^{2}\right), \\
2 W_{1}-f_{1} \sim \delta\left\{\gamma \sigma \zeta^{1 / 2}+\gamma \bar{\sigma}\left[(\gamma-1) C-\left(\gamma-1+2 \gamma^{*}\right)\right] \frac{1}{\zeta^{1 / 2}}+\zeta^{1 / 2} \sum_{n=2}^{\infty} \frac{()_{n}}{\zeta^{n}}\right\}+O\left(\delta^{2}\right), \\
f_{2} \sim f+\delta\left\{(1-\gamma)(f+\bar{f})+(1-\gamma) \bar{\sigma} \frac{1}{\zeta^{1 / 2}}+\sum_{n=2}^{\infty} \frac{()_{n}}{\zeta^{n / 2}}\right\}+O\left(\delta^{2}\right), \\
2 W_{2}-f_{2} \sim \delta\left\{\sigma \zeta^{1 / 2}+(\gamma-1)(\sigma-\bar{\sigma})+(\gamma-1) C \bar{\sigma} \frac{1}{\zeta^{1 / 2}}+\sum_{n=2}^{\infty} \frac{()_{n}}{\zeta^{n / 2}}\right\}+O\left(\delta^{2}\right),
\end{gathered}
$$

where the generic symbol ()$_{n}$ denotes an expression depending on the explicit forms of the outer expansion, (3.1). Some of them may involve arbitrary constants such as $C$ given in (3.25). These constants can only be determined from matching.

The above expressions represent the properties of inner expansions, which will be derived in the following section, for $\zeta \rightarrow \infty$. It follows from (3.28) and (3.29) that

$$
f_{1}=\text { Holomorphic function of } \zeta
$$

$$
2 W_{1}-f_{1}=\zeta^{1 / 2} \text { [Holomorphic function of } \zeta \text { ] }{ }^{\text {as }} \zeta \rightarrow \infty
$$

a property that can be directly deduced from the traction-free condition. On the other hand, (3.30) and (3.31) indicate that both $f_{2}$ and $W_{2}$ involve powers of $\zeta^{-1 / 2}$ as $\zeta \rightarrow \infty$. This property cannot be directly conceived from any of the governing conditions and will be needed in the series representation to be constructed in the sequel.

4. Inner expansion. The independent variable is the boundary layer variable $\zeta$ defined by (2.21). Guided by (3.28)-(3.31), we shall seek the expansion of a generic unknown function $F(z)$ in the form (c.f. (3.1))

$$
F(z) \sim F^{*}(\zeta, \delta) \sim \sum_{n=0}^{\infty} \delta^{n} F_{n}^{*}(\zeta)
$$

where $F$ stands for any one of the four unknowns $W_{1}$ and $f_{c}$. Using $F^{*}(\zeta)$ to denote $F^{*}(\zeta, \delta)$ for simplicity, we obtain from $(2.7),(2.12)$, and (2.13) the governing system:

$$
\begin{aligned}
& W_{1}^{* \pm}(\xi)+W_{1}^{* \mp}(\xi)-f_{1}^{* \mp}(\xi)=0 \quad(\xi<0) \\
& W_{2}^{*}\left(\zeta_{c}\right)+W_{2}^{*}\left(\bar{\zeta}_{c}\right)+\left(\zeta_{c}-\bar{\zeta}_{c}\right) \overline{W_{2}^{*} \prime\left(\zeta_{c}\right)}-f_{2}^{*}\left(\bar{\zeta}_{c}\right)
\end{aligned}
$$

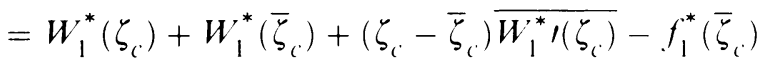

$$
\begin{aligned}
& W_{2}^{*}\left(\zeta_{c}\right)=\frac{1}{\gamma} W_{1}^{*}\left(\zeta_{c}\right)-\frac{\gamma^{*}}{\gamma}\left[W_{1}^{*}\left(\zeta_{c}\right)+W_{1}^{*}\left(\bar{\zeta}_{i}\right)+\left(\zeta_{c}-\bar{\zeta}_{i}\right) \overline{W_{1}^{* *}\left(\zeta_{i}\right)}-f_{1}^{*}\left(\bar{\zeta}_{c}\right)\right] .
\end{aligned}
$$


where $\zeta_{c}$ is defined by (2.22). As $\zeta \rightarrow \infty$, the functions must be matched with the inner expansions of the outer expansions (3.28)-(3.31).

The $\delta^{n}$-order conditions may be derived by using the following substitution:

$$
\begin{aligned}
F^{*}\left(\zeta_{c}\right) & \sim \sum \delta^{n} F_{n}^{*}\left(\zeta_{c}\right) \\
& \sim \sum \delta^{n}\left[F_{n}^{*}\left(\zeta_{0}\right) \mp \delta^{2} i \frac{1}{4}(1-\xi)^{3 / 2} F_{n}^{* \prime}\left(\zeta_{0}\right)+\cdots\right]
\end{aligned}
$$

where $\zeta_{0}$ is defined by (2.23). For the $\delta^{0}$-terms, the choice

$$
W_{10}^{*}(\zeta)=\frac{1}{2} f_{10}^{*}(\zeta)=\gamma W_{20}^{*}(\zeta)=\frac{\gamma}{2} f_{20}^{*}(\zeta)=\frac{\gamma}{2} f
$$

satisfies all the conditions and yields no useful information. The $\delta$-order system governs the desired asymptotic limit $(\delta \rightarrow 0)$ and the subsequent discussions will be concentrated on the solution of the system.

We begin by writing

$$
\begin{gathered}
f_{11}^{*}(\zeta)=\gamma\left[(1-\gamma)(f+\bar{f})-\left(1-\gamma-2 \gamma^{*}\right)(\sigma-\bar{\sigma})\right]+\gamma \sigma h_{1}(\zeta), \\
2 W_{11}^{*}(\zeta)-f_{11}^{*}(\zeta)=\gamma \sigma \zeta^{1 / 2}\left[1+H_{1}(\zeta)\right], \\
f_{21}^{*}(\zeta)=(1-\gamma)(f+\bar{f})+\sigma h_{2}(\zeta), \\
W_{21}^{*}(\zeta)=\frac{1}{2}(1-\gamma)[(f+\bar{f})-(\sigma-\bar{\sigma})]+\frac{\sigma}{2} \zeta^{1 / 2}+\sigma H_{2}(\zeta) .
\end{gathered}
$$

Comparing the above with $(3.28)-(3.31)$, we conclude that the explicitly listed terms are matched and

$$
h_{c r}(\zeta), \quad H_{r}(\zeta)=0 \quad \text { as } \zeta \rightarrow \infty .
$$

Since the geometry is symmetric and the loading symmetry is maintained by the factor $\sigma$ defined by (2.10), we conclude from the above that the functions $h_{\alpha}$ and $H_{r}$, signified by a generic symbol $F$, must satisfy the condition

$$
\overline{F(\zeta)}=F(\bar{\zeta}) \text { for } h_{c} \text { and } H_{1 \imath} \text {. }
$$

Finally, (4.2) is identically satisfied if $h_{1}$ and $H_{1}$ are holomorphic in $D_{1}$ in the $\zeta$-plane. We must now determine $h_{c r}$ and $H_{c r}$ to meet the two remaining conditions (4.3) and (4.4).

Before proceeding, we establish a few identities associated with the function $\zeta_{0}$ given by (2.23). Since

$$
\zeta_{0}^{1 / 2}=1+i \eta
$$

we may express $\bar{\zeta}_{0}^{1 / 2}$ in terms of $\zeta_{0}^{1 / 2}$, i.e.,

$$
\bar{\zeta}_{0}^{1 / 2}=\Psi\left(\zeta_{0}\right)=2-\zeta_{0}^{1 / 2}
$$

where $\Psi(\zeta)$ is holomorphic in the $\zeta\left(=\zeta_{1}+i \zeta_{2}\right)$-plane with a cut along the 
$-\zeta_{1}$ axis, the crack boundary. We have

$$
\begin{gathered}
\Psi\left(\Psi^{2}(\zeta)\right)=\zeta^{1 / 2}, \\
1 / \Psi(\zeta)=-\frac{4}{\zeta-4} \quad \text { as } \zeta \rightarrow 4, \\
\frac{1}{4} \frac{\zeta_{0}-\bar{\zeta}_{0}}{\bar{\zeta}_{0}^{1 / 2}}=\frac{1}{\Psi\left(\zeta_{0}\right)}-1 .
\end{gathered}
$$

Substituting (4.7), (4.8), and (4.10) into (4.4) and using (4.12), (4.14), and (4.17), we find that the condition is satisfied if

$$
\begin{aligned}
H_{2}(\zeta)= & H_{2}^{*}\left\{H_{1}, h_{1}\right\} \\
= & \frac{1}{2}\left[\zeta^{1 / 2} H_{1}(\zeta)+h_{1}(\zeta)\right] \\
& -\gamma^{*}\left\{\frac{1}{2}\left[h_{1}(\zeta)-h_{1}\left(\Psi^{2}(\zeta)\right)+\zeta^{1 / 2} H_{1}(\zeta)+\Psi(\zeta) H_{1}\left(\Psi^{2}(\zeta)\right)\right]\right. \\
& \left.+\sigma^{*}\left(\zeta^{1 / 2}-1\right)\left[\frac{H_{1}\left(\Psi^{2}(\zeta)\right)}{\Psi(\zeta)}+2 \Psi(\zeta) H_{1}^{\prime}\left(\Psi^{2}(\zeta)\right)+2 h_{1}^{\prime}\left(\Psi^{2}(\zeta)\right)\right]+\frac{\sigma^{*}}{\Psi(\zeta)}\right\}
\end{aligned}
$$

where

$$
\sigma^{*}=\frac{\bar{\sigma}}{\sigma}= \begin{cases}+1 & \text { if } \sigma=\sigma_{22} \\ -1 & \text { if } \sigma=i \sigma_{12}\end{cases}
$$

Substituting the above into (4.3) and using (4.14) and (4.15), we find that the condition is satisfied if

$$
\begin{aligned}
h_{2}(\zeta)= & h_{2}^{*}\left\{H_{1}, h_{1}\right\} \\
= & H_{2}(\zeta)-4 \sigma^{*}\left(\zeta^{1 / 2}-1\right) H_{2}^{\prime}(\zeta) \\
& +\frac{1}{2}\left(1-\gamma-\gamma^{*}\right)\left[\frac{2 \sigma^{*}}{\zeta^{1 / 2}}+\Psi(\zeta) H_{1}\left(\Psi^{2}(\zeta)\right)+h_{1}\left(\Psi^{2}(\zeta)\right)\right] \\
& -\left(\gamma+\gamma^{*}\right)\left\{\frac{1}{2}\left[\zeta^{1 / 2} H_{1}(\zeta)-h_{1}(\zeta)\right]\right. \\
& \left.+\sigma^{*}\left(1-\zeta^{1 / 2}\right)\left[\frac{H_{1}(\zeta)}{\zeta^{1 / 2}}+2 \zeta^{1 / 2} H_{1}^{\prime}(\zeta)+2 h_{1}^{\prime}(\zeta)\right]\right\}
\end{aligned}
$$

The above two expressions $H_{2}^{*}\left\{H_{1}, h_{1}\right\}$ and $h_{2}^{*}\left\{H_{1}, h_{1}\right\}$ may be taken as two calculating machines which convert the input $h_{1}$ and $H_{1}$ into the products $h_{2}$ and $H_{2}$ in such a way that the continuity conditions are identically satisfied. For a pair of chosen $H_{1}$ and $h_{1}$ satisfying (4.11), $H_{2}$ and $h_{2}$ calculated from (4.19) and (4.20) also satisfy (4.11). The only condition that is left to be enforced is that $h_{\alpha}$ and $H_{\alpha}$ must be holomorphic in $D_{\alpha}$ in the $\zeta$-plane (c.f. Fig. 1b). 
Let us begin by setting $H_{1}=h_{1}=0$. Then,

$$
\begin{aligned}
& H_{2}^{*}\{0,0\}=-\frac{\gamma^{*} \sigma^{*}}{\Psi(\zeta)} \\
& h_{2}^{*}\{0,0\}=\frac{\left(1-\gamma-\gamma^{*}\right) \sigma^{*}}{\zeta^{1 / 2}}-\frac{\gamma^{*} \sigma^{*}}{\Psi(\zeta)}+\frac{2 \gamma^{*}\left(\zeta^{1 / 2}-1\right)}{\zeta^{1 / 2}\left(2-\zeta^{1 / 2}\right)^{2}}
\end{aligned}
$$

which have poles at $\zeta=4$, i.e.,

$$
\begin{aligned}
& H_{2}^{*}\{0,0\}=P_{20}^{*}(\zeta)=\frac{4 \gamma^{*} \sigma^{*}}{\zeta-4} \quad(\zeta \rightarrow 4) \\
& h_{2}^{*}\{0,0\}=P_{20}^{*}(\zeta)=\frac{4 \gamma^{*} \sigma^{*}}{\zeta-4}+\frac{16 \gamma^{*}}{(\zeta-4)^{2}} \quad(\zeta \rightarrow 4) .
\end{aligned}
$$

Thus, $H_{1}=h_{1}=0$ cannot be the correct choice. To obtain the complete solution, we write

$$
\begin{aligned}
& H_{1}(\zeta)=R_{10}(\zeta) \\
& h_{1}(\zeta)=r_{10}(\zeta) \\
& H_{2}(\zeta)=H_{2}^{*}\{0,0\}+\left[R_{20}(\zeta)-P_{20}^{*}(\zeta)\right] \\
& h_{2}(\zeta)=h_{2}^{*}\{0,0\}+\left[r_{20}(\zeta)-p_{20}^{*}(\zeta)\right]
\end{aligned}
$$

where $R_{\alpha 0}$ and $r_{\alpha 0}$ are holomorphic in $D_{\alpha}$. The following series are assumed:

$$
\begin{aligned}
& r_{10}(\zeta)=\sum_{n=1}^{\infty} \frac{a_{n}}{(\zeta-4)^{n}}, \quad R_{10}=\sum_{n=1}^{\infty} \frac{b_{n}}{(\zeta-4)^{n}}, \\
& r_{20}(\zeta)=\sum_{n=1}^{\infty} \frac{A_{n}}{\zeta^{n / 2}}, \quad R_{20}=\sum_{n=1}^{\infty} \frac{B_{n}}{\zeta^{n / 2}},
\end{aligned}
$$

which conform with the requirement at $\zeta=\infty$ characterized by (3.28)-(3.31). The constants $a_{n}, b_{n}, A_{n}$, and $B_{n}$ must be chosen in such a way that the substitutions (c.f. $(4.7)-(4.10)$ )

$$
\begin{aligned}
& f_{1}^{*}: \gamma \sigma r_{10}(\zeta) \\
& 2 W_{1}^{*}-f_{1}^{*}: \gamma \sigma \zeta^{1 / 2} R_{10}(\zeta) \\
& f_{2}^{*}: \sigma\left[r_{20}(\zeta)-p_{20}^{*}(\zeta)\right] \\
& W_{2}^{*}: \sigma\left[R_{20}(\zeta)-P_{20}^{*}(\zeta)\right]
\end{aligned}
$$

satisfy the two continuity conditions (4.3) and (4.4). Since all terms are bounded, the standard Fast Fourier Transform Algorithm may be applied. This provides a numerical scheme for the direct computation of the needed asymptotic solution as opposed to the numerical extrapolation procedure reported by $\mathrm{Wu}$ and $\mathrm{Chen}$ [8]. The method may be applied to arbitrary inhomogeneity configurations and the results will be reported in a different paper.

Let $K_{I}$ and $K_{I I}$ be the SIF's. They may be normalized by the factors $\sigma_{22} \sqrt{\pi}$ and $\sigma_{12} \sqrt{\pi}$, i.e.,

$$
K_{1}=K_{I} / \sigma_{22} \sqrt{\pi}, \quad K_{2}=K_{I I} / \sigma_{12} \sqrt{\pi}
$$


where $K_{1}$ and $K_{2}$ depend on the small parameter $\delta$. As $\delta \rightarrow 0$, they tend to finite limits which may be obtained from (4.8). The results are

$$
\begin{array}{ll}
K_{1}=\gamma\left[1+H_{1}(0)\right] & \left(\sigma^{*}=\frac{\bar{\sigma}}{\sigma}=+1\right), \\
K_{2}=\gamma\left[1+H_{1}(0)\right] & \left(\sigma^{*}=\frac{\bar{\sigma}}{\sigma}=-1\right),
\end{array}
$$

where

$$
H_{1}(0)=R_{10}(0)=\sum_{n=1}^{\infty} \frac{b_{n}}{(-4)^{n}} .
$$

The rest of the section will be devoted to the determination of an approximate but explicit solution.

Another way of removing the poles defined by (4.23) and (4.24) is to define $H_{1}$ and $h_{1}$ as follows:

$$
\begin{aligned}
& H_{1}(\zeta)=P_{11}^{*}(\zeta)=P_{1}(\zeta)=\frac{P_{11}}{\zeta-4}+\frac{P_{12}}{(\zeta-4)^{2}} \\
& h_{1}(\zeta)=p_{11}^{*}(\zeta)=p_{1}(\zeta)=\frac{p_{11}}{\zeta-4}+\frac{p_{12}}{(\zeta-4)^{2}}
\end{aligned}
$$

where $P_{11}, P_{12}, p_{11}$, and $p_{12}$ are constants and both expressions are holomorphic in $D_{1}$ in the $\zeta$-plane.

Substituting the above into (4.18) and (4.20) and requiring that the calculated $\mathrm{H}_{2}$ and $h_{2}$ are free of poles at $\zeta=4$, we obtain

$$
\begin{aligned}
& 2 P_{1}(\zeta)+p_{1}(\zeta)+\frac{8 \gamma^{*} \sigma^{*}\left[1+H_{1}(0)\right]}{1-\gamma^{*}} \frac{1}{\zeta-4}=0, \\
& \left(2-\sigma^{*}\right) P_{1}(\zeta)-p_{1}(\zeta)-4\left[2 P_{1}^{\prime}(\zeta)+p_{1}^{\prime}(\zeta)\right]=0,
\end{aligned}
$$

and hence

$$
\begin{aligned}
& P_{1}(\zeta)=\frac{\Gamma}{4-\sigma^{*}}\left[-\frac{1}{\zeta-4}+\frac{4}{(\zeta-4)^{2}}\right] \\
& p_{1}(\zeta)=\frac{\Gamma}{4-\sigma^{*}}\left[\frac{\sigma^{*}-2}{\zeta-4}-\frac{8}{(\zeta-4)^{2}}\right]
\end{aligned}
$$

where

$$
\Gamma=\frac{8 \gamma^{*} \sigma^{*}}{1-\gamma^{*}}\left[1+H_{1}(0)\right]
$$

The newly generated $\mathrm{H}_{2}$ and $h_{2}$ are now free of poles at $\zeta=4$, but new poles are generated at $\zeta=16$. This is so because $H_{1}\left(\Psi^{2}(\zeta)\right)$ now include powers of

$$
\frac{1}{\Psi^{2}(\zeta)-4}=-\frac{2}{\zeta-16} \quad \text { as } \zeta \rightarrow 16
$$

Removing the poles at $\zeta=16$ will lead to new poles at $\zeta=36$. 
The process may be continued for a chosen number of times so that

$$
\begin{aligned}
& H_{1}=P_{1 N}^{*}(\zeta)=\sum_{n=1}^{N} P_{n}(\zeta), \\
& h_{1}=p_{1 N}^{*}(\zeta)=\sum_{n=1}^{n} p_{n}(\zeta),
\end{aligned}
$$

where

$$
\begin{aligned}
& p_{n}(\zeta)=\sum_{m=1}^{2 n} \frac{P_{n m}}{\left[\zeta-(2 n)^{2}\right]^{m}}, \\
& p_{n}(\zeta)=\sum_{m=1}^{2 n} \frac{p_{n m}}{\left[\zeta-(2 n)^{2}\right]^{m}},
\end{aligned}
$$

which may be expressed in terms of $P_{n-1}(\zeta)$ and $p_{n-1}(\zeta)$ via recurrence formulas. The derivation is straightforward but lengthy and is not included in this paper.

The functions generated by substituting (4.39) and (4.40) into (14.18) and (4.20) have poles at $\zeta=[2(N+1)]^{2}$, i.e.,

$$
\begin{aligned}
& H_{2}^{*}\left\{P_{1 N}^{*}(\zeta), p_{1 N}^{*}(\zeta)\right\}=P_{2 N}^{*}(\zeta) \\
& h_{2}^{*}\left\{P_{1 N}^{*}(\zeta), p_{1 N}^{*}(\zeta)\right\}=p_{2 N}^{*}(\zeta)
\end{aligned} \text { as } \zeta \rightarrow[2(N+1)]^{2}
$$

where $P_{2 N}^{*}$ and $p_{2 N}^{*}$ have forms similar to those given by (4.39) and (4.40). Thus, (4.39) and (4.40), together with the generated $\mathrm{H}_{2}$ and $h_{2}$, cannot be the correct solution. The complete solution must be of the form

$$
\begin{aligned}
& H_{1}(\zeta)=P_{1 N}^{*}(\zeta)+R_{1 N}(\zeta), \\
& h_{1}(\zeta)=p_{1 N}^{*}(\zeta)+r_{1 N}(\zeta), \\
& H_{2}(\zeta)=H_{2}^{*}\left\{P_{1 N}^{*}, p_{1 N}^{*}\right\}+\left[R_{2 N}(\zeta)-P_{2 N}^{*}(\zeta)\right], \\
& h_{2}(\zeta)=h_{2}^{*}\left\{P_{1 N}^{*}, p_{1 N}^{*}\right\}+\left[r_{2 N}(\zeta)-p_{2 N}^{*}(\zeta)\right],
\end{aligned}
$$

where $R_{\alpha N}$ and $r_{\alpha N}$ play the roles of $R_{\alpha 0}$ and $r_{\alpha 0}$ in (4.25) and must be determined in a similar manner. In fact, the series (4.26) may be taken as the sol tion for the new unknowns. The input to this problem is provided by $P_{2 N}^{*}$ and $p_{2 N}^{*}$ which have poles of order $2 N$ at $\zeta=[2(N+1)]^{2}$. Noting that the nearest interface point is at $\zeta=1$, we conclude that the contributions of $R_{\alpha N}$ and $r_{\alpha N}$ are perhaps small for large $N$. This, however, is not proven in this paper ${ }^{\dagger}$. Taking the statement for granted, we have

$$
\begin{aligned}
& H_{1}(\zeta) \approx P_{1 N}^{*}(\zeta), \quad h_{1}(\zeta) \approx p_{1 N}^{*}(\zeta) \\
& H_{2}(\zeta) \approx H_{2}^{*}\left\{P_{1 N}^{*}, P_{1 N}^{*}\right\}-P_{2 N}^{*}(\zeta), \\
& h_{2}(\zeta) \approx h_{2}^{*}\left\{P_{1 N}^{*}, p_{1 N}^{*}\right\}-p_{2 N}^{*}(\zeta) .
\end{aligned}
$$

\footnotetext{
${ }^{\dagger}$ The recurrence formulas associated with (4.41) and (4.42), together with the explicit forms of (4.43), are needed for such a proof.
} 
For a two-term approximation, $P_{1}$ and $p_{1}$ are given by (4.35) and (4.36). A lengthy computation yields the following expressions for $P_{2}$ and $p_{2}$ :

$$
\begin{aligned}
& P_{2}(\zeta)=\frac{2 \Gamma}{\left(4-\sigma^{*}\right)\left(16-3 \sigma^{*}\right)}\{ {\left[\frac{2\left(1-\gamma-\gamma^{*}\right) \sigma^{*}}{\gamma+\gamma^{*}}+\frac{\left.4\left(2+\sigma^{*}\right) \gamma^{*}\right]}{1-\gamma^{*}}\right] \frac{1}{(\zeta-16)} } \\
&-\left[\frac{64\left(1-\gamma-\gamma^{*}\right)}{\gamma+\gamma^{*}}+\frac{48 \gamma^{*}\left(2+3 \sigma^{*}\right)}{1-\gamma^{*}}\right] \frac{1}{(\zeta-16)^{2}} \\
&+\frac{384 \gamma^{*}\left(3+11 \sigma^{*}\right)}{1-\gamma^{*}} \frac{1}{(\zeta-16)^{3}} \\
&\left.-\frac{(3 \cdot 16)^{3} \gamma^{*}}{1-\gamma^{*}} \frac{1}{(\zeta-16)^{4}}\right\} \\
& p_{2}(\zeta)=-\frac{3 \sigma^{*}}{4} P_{2}(\zeta) \quad \frac{\Gamma}{2\left(4-\sigma^{*}\right)}\left\{\left[-\frac{2\left(1-\gamma-\gamma^{*}\right) \sigma^{*}}{\gamma+\gamma^{*}}+\frac{4\left(2+\sigma^{*}\right) \gamma^{*}}{1-\gamma^{*}}\right] \frac{1}{(\zeta-16)}\right. \\
&+ {\left[\frac{64\left(1-\gamma-\gamma^{*}\right)}{\gamma+\gamma^{*}}+\frac{48 \gamma^{*} \sigma^{*}}{1-\gamma^{*}}\right] \frac{1}{(\zeta-16)^{2}} } \\
&+ \frac{384 \gamma^{*}\left(11 \sigma^{*}-3\right)}{1-\gamma^{*}} \frac{1}{(\zeta-16)^{3}} \\
&\left.+\frac{(3 \cdot 16)^{3} \gamma^{*}}{1-\gamma^{*}} \frac{1}{(\zeta-16)^{4}}\right\}
\end{aligned}
$$

where $\Gamma$ is defined by (4.37). It follows from the approximation

$$
H_{1}(0) \approx P_{1}(0)+P_{2}(0)
$$

that

$$
\begin{aligned}
& \frac{H_{1}(0)}{1+H_{1}(0)} \approx \frac{\gamma^{*} \sigma^{*}}{\left(1-\gamma^{*}\right)\left(4-\sigma^{*}\right)}\left\{4-\frac{1}{16-3 \sigma^{*}}\left[\frac{2\left(2+\sigma^{*}\right)\left(1-\gamma-\gamma^{*}\right)}{\gamma+\gamma^{*}}\right.\right. \\
& \left.\left.+\frac{\left(91+59 \sigma^{*}\right) \gamma^{*}}{2\left(1-\gamma^{*}\right)}\right]\right\}
\end{aligned}
$$

The approximate expressions for the asymptotic values of $K_{1}$ and $K_{2},(4.29)$, are

$$
\begin{aligned}
& K_{1} \simeq \frac{3 \gamma\left(1-\gamma^{*}\right)}{3-7 \gamma^{*}+\frac{\nu^{*}}{13}\left[\frac{6\left(1-\gamma-\gamma^{*}\right)}{\gamma+\gamma^{*}}+\frac{75 \gamma^{*}}{1-\gamma^{*}}\right]}, \\
& K_{2} \simeq \frac{5 \gamma\left(1-\gamma^{*}\right)}{5-\gamma^{*}-\frac{\check{\nu}^{*}}{19}\left[\frac{2\left(1-\gamma-\gamma^{*}\right)}{\gamma+\gamma^{*}}+\frac{16 \gamma^{*}}{1-\gamma^{*}}\right]} .
\end{aligned}
$$

5. Results and discussion. We shall restrict our attention to the range $0<\mu_{1} / \mu_{2}<$ 1 to reflect our interest in the physical situation of crack damage interaction. The confocal nature of the title problem allows a series solution in a transformed plane. Such a series solution was obtained by $\mathrm{Wu}$ and Chen [8]. The convergence of the needed matrix inversion becomes extremely slow for $\varepsilon<0.05$. Nevertheless, the 


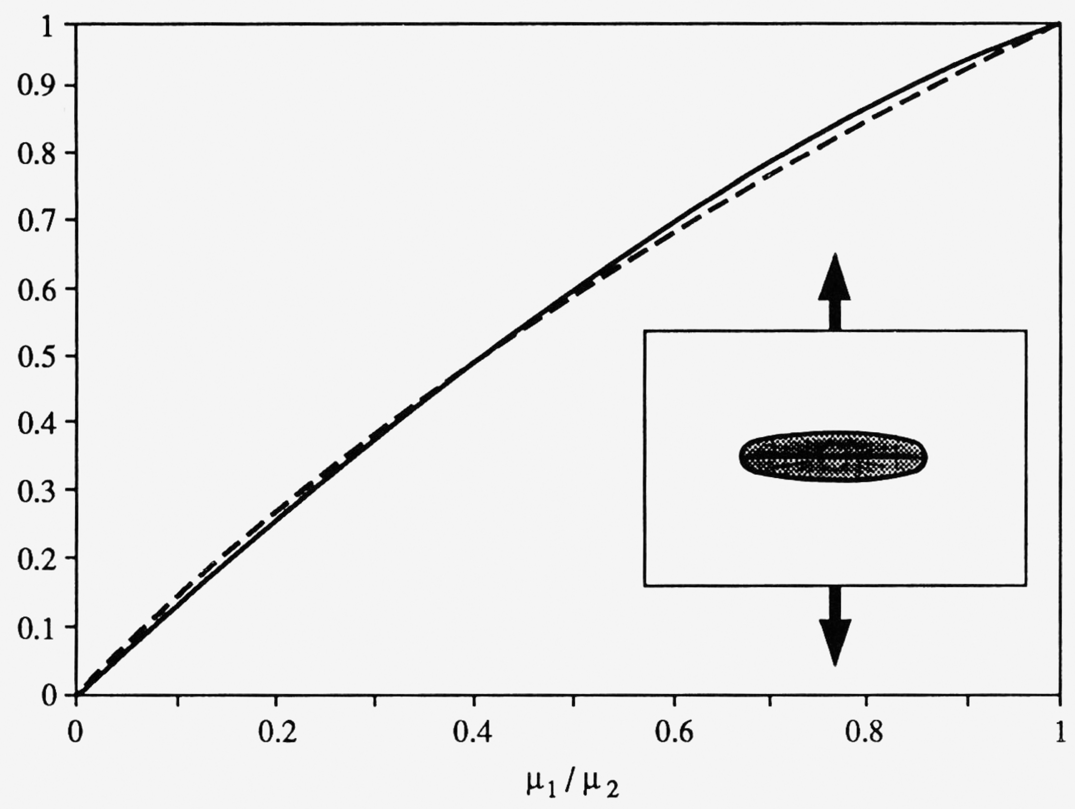

FIG. 2. $K_{1}$ as a function of $\mu_{1} / \mu_{2}$ for $b / a \quad 0$ and $\nu_{1}=\nu_{2}=0.2$ (plane strain). The dashed line is the numerical result of [8] and the solid line is the two-term approximate formula (4.50).

$\varepsilon \rightarrow 0$ limit was accurately extrapolated from the extensive numerical results. This limit is reproduced for $K_{1}$ for the case $\nu_{1}=\nu_{2}=0.2$ in Fig. 2. The accuracy of our two-term approximate solution, (4.50) and (4.51), is also demonstrated in Fig. 2.

To substantiate our claim that the same result may be directly computed from the series solution (4.25)-(4.27), a readily available Fast Fourier Transform routine was applied to the system with the series truncated at $n=10$, and the directly computed $K_{1}$ is almost indistinguishable from the extrapolated result for the case given in Fig. 2. It should be emphasized that the series solution not only gives the numerically exact SIF's but is actually the numerically exact field solution which may be used to compute the Eshelby force along the interface. In particular, the energy release rate associated with an inhomogeneity-front propagation may be computed. This is the real usefulness of the desired series solution. Our analysis has established the fact that the correct series representation for the matrix in the neighborhood of a crack tip must have the properties of $r_{20}$ and $R_{20}$ given by (4.26). The adaptation of the series representation to a general inhomogeneity configuration, together with the aforementioned front-propagation calculation, is the main objective of our intention.

Our formulation indicates that the solution to the title problem depends explicitly on the two composite parameters $\gamma$ and $\gamma^{*}$ defined by (2.14). It is therefore desirable to present the SIF's in terms of them. The choice of the two composite parameters in a typical two-phase problem is not unique (Dundurs [1]). The parameters $\gamma$ and $\gamma^{*}$ appear naturally in our problem. Moreover, exact solution may be obtained for 


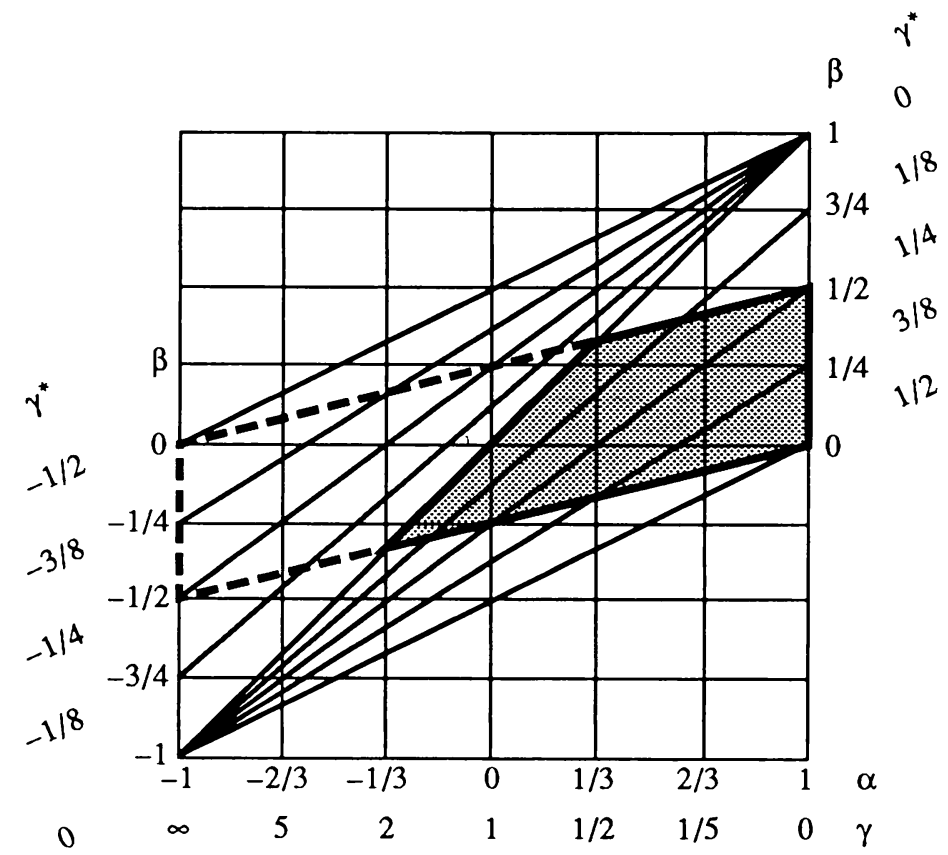

FIG. 3. Admissible region in terms of composite elastic constants $(\alpha, \beta)$ and $\left(\gamma, \gamma^{*}\right)$. The shaded region is for $\mu_{1} / \mu_{2}<1$.

the case $\gamma^{*}=0$ (Wu and Chen [8]). Still, the range of the two parameters may be easily determined from Dundurs' discussion. In terms of $\gamma$ and $\gamma^{*}$, Dundurs' $\alpha$ and $\beta$ parameters are

$$
\alpha=\frac{1-\gamma}{1+\gamma}, \quad \beta=\frac{1-\gamma}{1+\gamma}-\frac{2 \gamma^{*}}{1+\gamma},
$$

which may be inverted to yield

$$
\gamma=\frac{1-\alpha}{1+\alpha}, \quad \gamma^{*}=\frac{\alpha-\beta}{1+\alpha} .
$$

Dundurs' $\alpha \beta$-plane, together with the admissible ranges of $\alpha$ and $\beta$, are reproduced in Fig. 3. Also plotted in the figure are the constant $\gamma$ and $\gamma^{*}$ lines. Thus, the ranges of $\gamma$ and $\gamma^{*}$ may be straightforwardly calculated, i.e.,

$$
\begin{array}{rr}
\frac{1}{2}\left(1-4 \gamma^{*}\right)<\gamma<2\left(1-2 \gamma^{*}\right) & \text { for } 0 \leq \gamma^{*} \leq \frac{1}{4} \\
0<\gamma<2\left(1-2 \gamma^{*}\right) & \text { for } \frac{1}{4} \leq \gamma^{*} \leq \frac{1}{2}
\end{array}
$$

which covers the range of interest set by $0 \leq \mu_{1} / \mu_{2} \leq 1$. Results pertaining to (4.50) and (4.51) are plotted in Fig. 4 as functions of $\gamma$ with $\gamma^{*}$ as a parameter. It is felt 


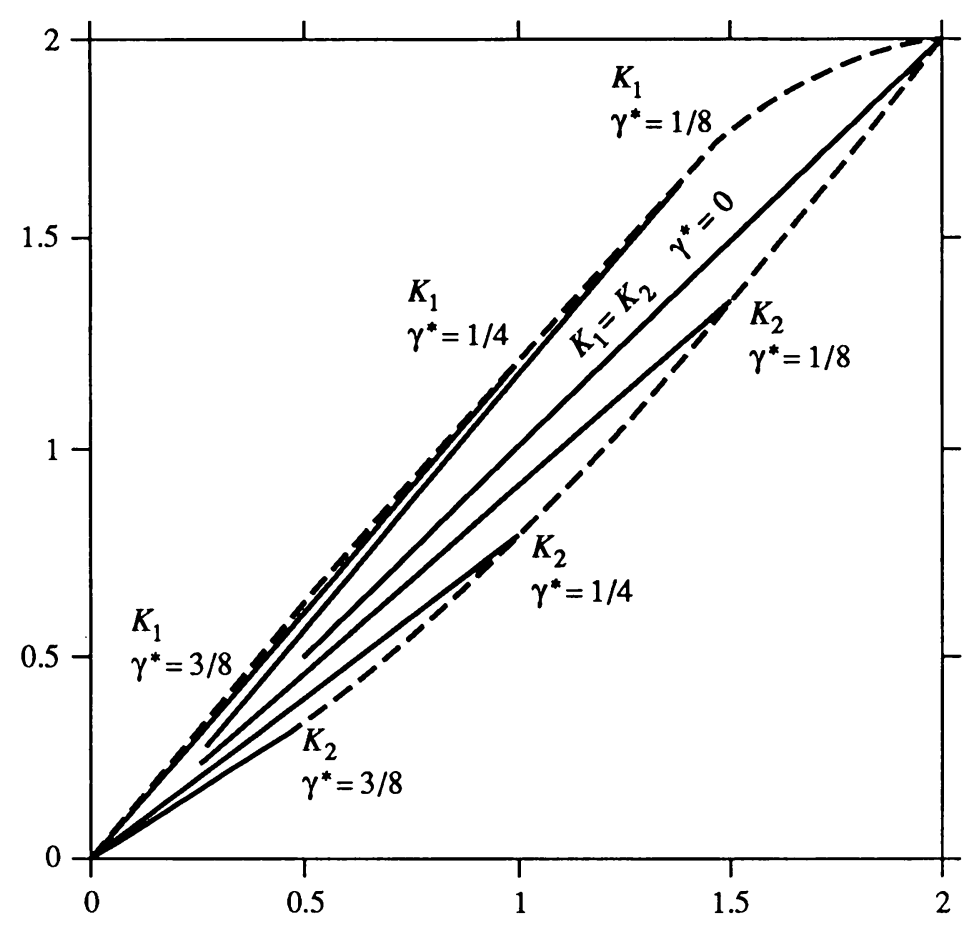

FIG. 4. $K_{1}$ and $K_{2}$ for all admissible combinations of $\gamma$ and $\gamma^{*}$.

that results for all inhomogeneity problems should be presented in terms of $\gamma$ and $\gamma^{*}$ in the fashion characterized by Fig. 4.

\section{REFERENCES}

[1] J. Dundurs, Edge-bonded dissimilar orthogonal elastic wedges under normal and shear loading -discussion, J. Appl. Mech. 36, 650-652 (1969)

[2] A. H. England, Complex Variable Method in Elasticity, Wiley-Interscience, New York, 1971

[3] J. D. Eshelby, The force on an elastic singularity, Philos. Trans. Roy. Soc. London A 224, 87-112 (1951)

[4] J. W. Hutchinson, Crack Tip Shielding by Micro-Cracking in Brittle Solids, Mech-87, Harvard University, 1986

[5] P. S. Steif, A semi-infinite crack partially penetrating a circular inclusion, J. Appl. Mech. 54, 87-92 (1987)

[6] M. Van Dyke, Perturbation Methods in Fluid Mechanics, Parabolic Press, Stanford, 1975

[7] C. H. Wu, A Semi-infinite crack penetrating an inclusion, J. Appl. Mech. 55, 736-738 (1988)

[8] C. H. Wu and C. H. Chen, $A$ crack in a confocal elliptic inhomogeneity embeded in an infinite medium, J. Appl. Mech., to appear 